\title{
Extended Adjuvant Chemotherapy in Triple-Negative Breast Cancer
}

\author{
Marzia A. Locatellia ${ }^{a}$ Giuseppe Curigliano $^{a} \quad$ Alexandru Eniu $^{b}$ \\ ${ }^{a}$ New Drugs Development Division, European Institute of Oncology, Milan, Italy; \\ ${ }^{\text {b } C a n c e r ~ I n s t i t u t e ~ I o n ~ C h i r i c u t a, ~ C l u j-N a p o c a, ~ R o m a n i a ~}$
}

\section{Keywords}

Triple-negative breast cancer - Adjuvant chemotherapy . Duration of adjuvant therapy .

Metronomic chemotherapy · Biologic agents

\section{Summary}

Triple-negative breast cancer (TNBC) represents a heterogeneous breast cancer subtype with a poor prognosis. The optimal adjuvant chemotherapy regimen is still unknown. Although numerous large randomized trials have established the benefit of adjuvant anthracyclines and/or taxanes in TNBC, there is no preferred regimen for these patients. There is currently no guideline. Moreover, without knowing the optimal treatment backbone, it will not be possible to evaluate whether adding agents such as platinum or other novel therapies is beneficial for TNBC patients. Furthermore, the best duration of adjuvant treatment in TNBC is still unknown. This review will focus on results of clinical trials that analyzed the benefits of extending the duration of adjuvant treatment in TNBCs with maintenance treatments. We will further discuss promising results in favor of other new agents including capecitabine, metronomic treatment, and biological drugs.

(c) 2017 S. Karger GmbH, Freiburg

\section{Introduction}

Triple-negative breast cancer (TNBC) is defined by $\leq 1 \%$ expression of the estrogen receptor (ER) and progesterone receptor, and normal human epidermal growth factor receptor 2 (HER2) gene copy number and expression. TNBC accounts for $12-17 \%$ of all breast cancers (BC). TNBC has shown an increased rate of BC- related deaths within the first 5 years. Additionally, once metastatic, patients with TNBC have shorter overall survival (OS) in comparison to those with other subtypes. TNBC patients are prone to developing visceral metastases more often than bone metastases when compared to other subtypes. Generally, TNBC occurs in younger women. Despite the greater relative benefit of chemotherapy (CT) in this patient population and a large number of clinical trials in the last decade, no optimal neo/adjuvant CT regimen has been identified [1-4].

\section{Heterogeneity in TNBC}

TNBC has a significant variability in pathological and morphological features with many potential molecular targets. Lehmann et al. [5] have proposed one of the most frequently used subclassifications, which includes 4 subtypes: 2 basal-like (BL1 and BL2), a mesenchymal, and a luminal androgen receptor subtype.

Approximately $70 \%$ of TNBCs contain $20 \%$ or more tumor-infiltrating lymphocytes (TILs) in the tumor stroma [6]. The generally higher level of TILs and the higher expression of immune checkpoint molecules in TNBC compared with ER-positive (ER+) BCs also makes TNBC an attractive target for immunotherapy. Immune-rich TBNC has a more favorable prognosis.

Androgen receptor-positive TNBC accounts for approximately $10 \%$ of all TNBCs and tends to have a more indolent course and share certain features with $\mathrm{ER}+\mathrm{BCs}$, including expression of several estrogen-regulated genes and frequent PIK3CA mutations. Anti-androgens are being investigated as a potential treatment in this subset.

Germline BRCA-mutant TNBCs represent another potential therapeutically relevant subtype. Germline BRCA1 and BRCA2 mutations are more frequent in TNBC than in other BC subtypes. There is increasing evidence that germline BRCA-mutant BCs have above-average platinum sensitivity and increased sensitivity to poly(ADP-ribose) polymerase (PARP) inhibitors.

\section{KARGER}

() 2017 S. Karger GmbH, Freiburg

Fax +497614520714 


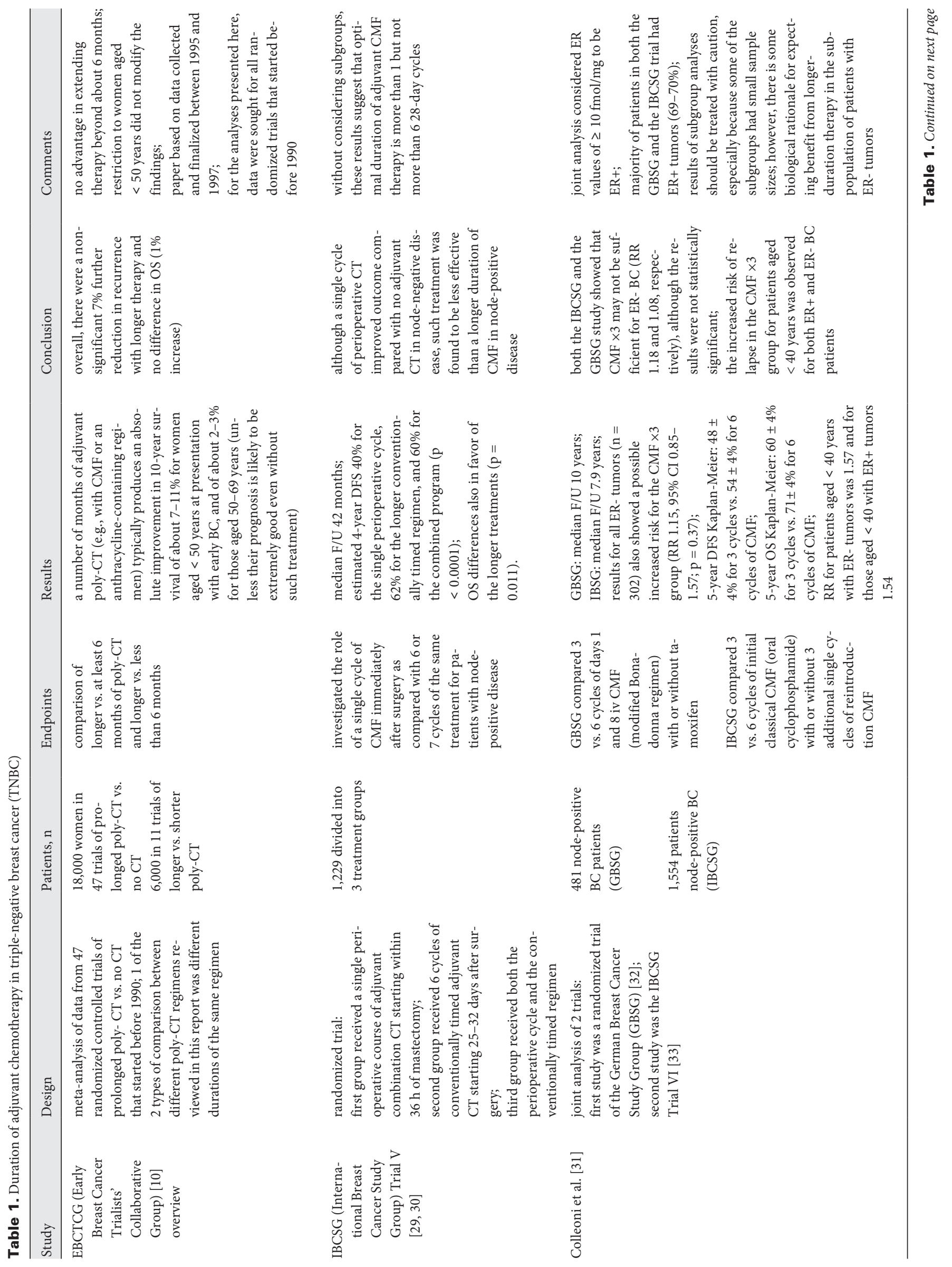




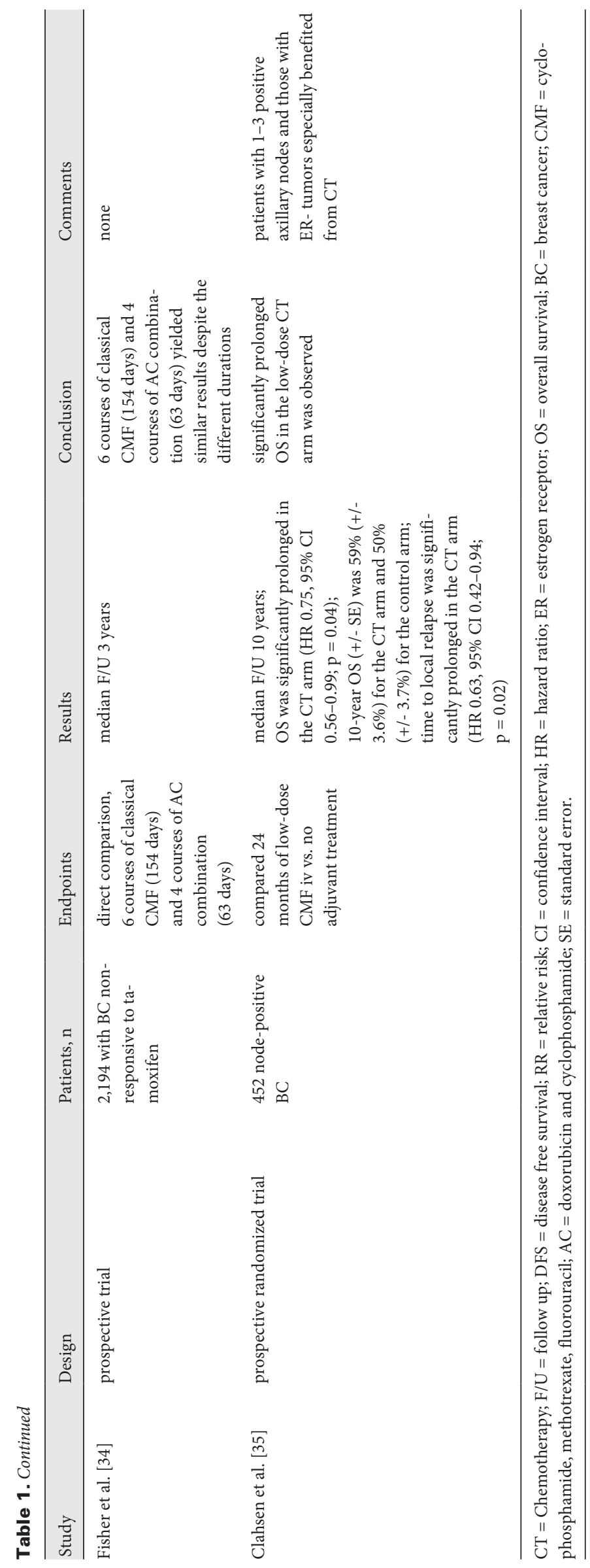

\section{Adjuvant Therapies for Early-Stage TNBC}

The optimal adjuvant CT regimen is still unknown. A 6-9 times higher risk for relapse has been reported for patients with TNBC who do not achieve a pathologic complete response (pCR) with neoadjuvant treatment. Because of this strong prognostic effect, pCR was proposed as a surrogate for OS in these 2 BC subtypes [7-9]. Numerous clinical trials have been designed to test the value of further adjuvant therapy in TNBC patients with no pCR. Although numerous large randomized trials have established the benefit of adjuvant anthracyclines and/or taxanes in TNBC, there is no preferred regimen for these patients, unlike for those with other BC subtypes. There is currently no guideline. Moreover, without knowing the optimal treatment backbone, it will not be possible to evaluate whether adding agents such as platinum or other novel therapies is beneficial for TNBC patients. There are promising results in favor of other new agents including capecitabine (X), platinum-based agents, and ixabepilone.

\section{Duration of Adjuvant Chemotherapy in TNBC}

Several trials have addressed the question of the optimal duration of adjuvant CT for BC patients. Duration of CT might be best tested in patients with ER-negative (ER-) tumors, where cytotoxic rather than endocrine effects of CT might prevail. Although this is a small subset compared with the entire BC population, it might represent a group with particular relevance for specific treatment analysis pertaining to questions of adjuvant CT.

Several trials are examining regimens that differ in duration of therapy but also in the drugs given. In these trials, the effects of treatment duration and drug choice are inextricably confounded. Differing conclusions about treatment duration are being reached.

CMF (cyclophosphamide, methotrexate, and fluorouracil) regimens were widely used in the 1990s, and were followed by the introduction of anthracycline-containing regimens and anthracycline/taxane regimens as suitable options for TNBC patients. In 1998, the Early Breast Cancer Trialists' Collaborative Group (EBCTCG) [10] presented a meta-analysis of data from 47 randomized controlled trials of prolonged poly-CT versus no CT that started before 1990. 1 of the 2 types of comparison between different poly-CT regimens reviewed in this report was different durations of the same regimen. The analysis involved 6,104 women in 11 trials and evaluated the comparison of longer versus at least 6 months of poly-CT and longer versus less than 6 months. Overall, there were a non-significant $7 \%$ further reduction in recurrence with longer therapy and no difference in OS (1\% increase). The restriction to women aged $<50$ years did not modify these findings.

Table 1 summarizes the results of the most significant trials which addressed the question of the optimal duration of adjuvant CT.

The US Intergroup trial investigating the addition of 4 cycles of paclitaxel following 4 cycles of AC (cyclophosphamide, doxorubicin) demonstrated a small but significant improvement in disease-free survival (DFS) and OS for the longer adjusted regimen [11]. This improvement was observed almost exclusively among patients with ER- tumors who did not receive tamoxifen. 


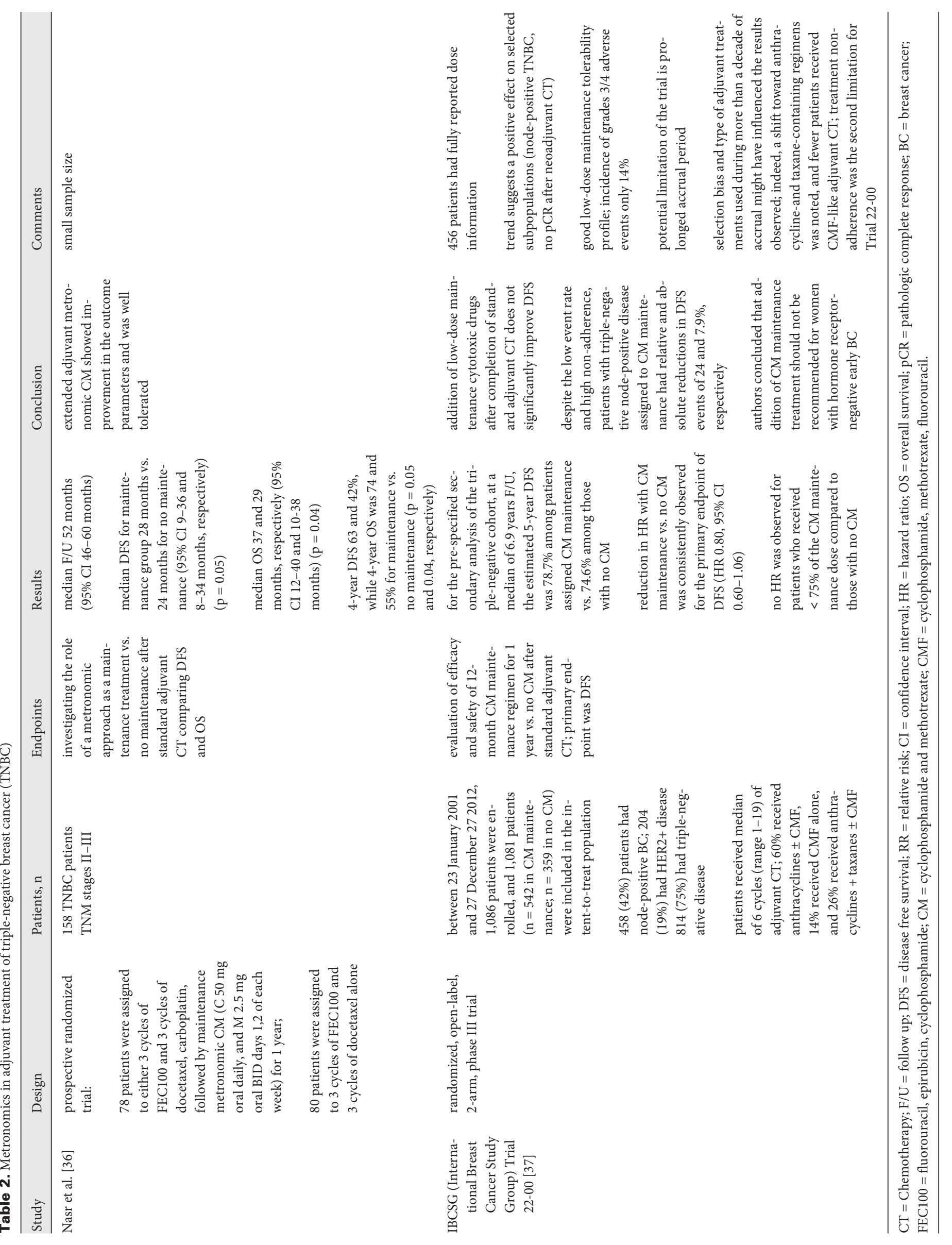


Hence, there are conflicting results on antitumor activity for CT administered for a prolonged time.

\section{Metronomics in the Adjuvant Treatment of TNBC}

The term metronomic CT was first introduced almost 20 years ago by Douglas Hanahan following the results of a preclinical study published by Jutah Folkman and Nob Kerbel $[12,13]$.

Chronically administered low-dose cyclophosphamide produces apoptosis of endothelial cells in the tumor microvasculature with a compromised repair process, which induces a prolonged antiangiogenic effect [12]. Low-dose methotrexate (M) inhibits endothelial cell proliferation in vitro and blocks endothelial cell growth factorinduced neovascularization in the rabbit cornea assay [14].

In the past 15 years, metronomic CT demonstrated clinical activity associated with a low incidence of adverse events (AEs) in patients with metastatic BC [15]. In 2014, André et al. [16] showed that metronomic CT has an immune-stimulatory effect through different mechanisms and an effect on tumor-initiating cells and on tumor dormancy, and might finally also interfere with the clonal evolution of tumors.

The antiangiogenic and pro-immune nature of metronomic CT makes TNBC a good candidate for re-induction of tumor dormancy or eradication of residual cancer cells [17].

Table 2 summarizes the results of the principal studies that investigated the role of a metronomic approach as maintenance treatment versus no maintenance after standard adjuvant CT in TNBC patients.

\section{Capecitabine in the Adjuvant Treatment of TNBC}

Several ongoing trials are addressing the intensification of adjuvant $\mathrm{CT}$ in TNBC patients, either through integration of novel agents into the adjuvant setting such as platinum, $\mathrm{X}$, ixabepilone, or bevacizumab, or through introduction of maintenance therapy such as X (CIBOMA and SYSUCC-001) or bevacizumab (BEATRICE).

Preliminary results of the CREATE-X (JBCRG-04) trial by the Japan Breast Cancer Research Group were presented at the 2015 San Antonio Breast Cancer Symposium [18]. The trial randomized 910 patients to observation versus 8 cycles of $\mathrm{X}$ therapy, and reported improved rates of 2 -year DFS ( 87.3 vs. $80.5 \%$; $\mathrm{p}=0.001$ ) and OS ( 96.2 vs. $93.9 \%$; $\mathrm{p}=0.086$ ) with X. All of the observed benefit was driven by the improved outcome in the ER- subpopulation ( $n=296)$ of the study.

Chen et al. [19] evaluated the clinical value of X-based combination adjuvant $\mathrm{CT}$ in early $\mathrm{BC}(\mathrm{EBC})$. The authors analyzed 8 randomized controlled trials with 14,072 participants. X-based combination adjuvant CT demonstrated a $16 \%$ increase in $\mathrm{BC}$-specific survival (BCSS) (hazard ratio (HR) 0.84, 95\% confidence interval (CI) $0.71-0.98 ; \mathrm{p}=0.03$ ) in the OS analysis and a $22 \%$ improvement in DFS (HR 0.78, 95\% CI 0.64-0.96; $\mathrm{p}=0.02$ ) in the hormone receptor-negative subgroup. However, there were no significant differences in DFS (HR 0.96, 95\% CI 0.89-1.05; $\mathrm{p}=0.38$ ), OS (HR 0.91, 95\% CI 0.82-1.00; $p=0.06$ ), or relapse between X-based and $\mathrm{X}$-free combination adjuvant CT. Regarding safety, reduced myelosuppression and hand-foot syndrome (HFS) development were observed in X-treated patients. X-based combination adjuvant CT might provide some BCSS benefit compared with X-free regimens in $\mathrm{EBC}$, but the absolute survival gain is small, and the survival benefit appears to be restricted to patients with hormone receptornegative EBC, which may indicate a target population for X-based combination adjuvant CT.

In 2015, Alagizy et al. [20], in a prospective phase II study, evaluated the tolerability and efficacy of metronomic $\mathrm{X}$ as an extended adjuvant treatment for women with TNBC. The trial enrolled 41 patients between June 2010 and December 2013. The patients received $500 \mathrm{mg}$ X orally BID continuously for 6 months after 6 cycles of adjuvant CT with FEC100 (fluorouracil, epirubicin, cyclophosphamide) \pm postoperative radiotherapy. The choice of giving uniform adjuvant CT to the patients enrolled allowed better assessment of the efficacy of metronomic X. The study showed that extended adjuvant metronomic $X$ was well tolerated with no grade 3 or 4 toxicities or life-threatening AEs at all. Only 6 (15\%) relapse events occurred. The incidence of both local and distant relapses was lower in comparison to other studies, but a larger sample and longer follow-up are required for better and more mature results. The effect of lowerdose X on DFS needs to be evaluated in a larger phase III study.

CIBOMA 2004-01/GEICAM 2003-11 [21] is a multicenter, open-label, randomized phase III trial evaluating the efficacy of maintenance treatment with $\mathrm{X}$ in operable TNBC node-positive patients (or node-negative with a tumor diameter of $\geq 1 \mathrm{~cm}$ ) who have received standard anthracyclines and or taxane-containing CT. Patients were randomized to Arm B (8 cycles of $1,000 \mathrm{mg} / \mathrm{m}^{2}$ X BID, days 1-14, q21d) or the observation (Arm B). The primary endpoint was DFS. The first safety data, presented at the 2010 San Antonio Cancer Symposium [22], revealed that the safety profile of adjuvant $\mathrm{X}$ as maintenance therapy was consistent with its known toxicity profile. More than $75 \%$ of patients were able to continue their treatment as planned with approximately $15 \%$ discontinuing due to toxicity or withdrawal. Grade 3/4 AEs were higher with maintenance X: HFS $17.4 \%$, diarrhea $2.9 \%$, vomiting $1.0 \%$, and elevated bilirubin $1.0 \%$.

SYSUCC-001 [23] is an open-label, randomized phase III trial evaluating the efficacy of adjuvant X metronomic CT in TNBC. In this study, which is still recruiting participants, patients with early TNBC are randomized to treatment with standard adjuvant CT alone or standard adjuvant CT followed by 1 year of $\mathrm{X}$ metronomic therapy $650 \mathrm{mg} / \mathrm{m}^{2}$ BID. No published data are yet available.

\section{Biological Agents in the Adjuvant Treatment of TNBC}

In another effort to evaluate the benefit of maintenance treatment for TNBC patients, the BEATRICE study [24] enrolled 1,290 patients to receive CT alone and 1,301 to receive bevacizumab (B) plus CT between 3 December 2007 and 8 March 2010. Most patients received anthracycline-containing therapy; 1,638 (63\%) of the 2,591 patients had node-negative BC. At the time of analysis for invasive DFS (IDFS), median follow-up was 31.5 months (interquartile range 25.6-36.8) in the CT alone group and 32.0 months (27.5-36.9) in the B group. The 3-year IDFS was $82.7 \%$ (95\% CI 80.5-85.0) with CT alone and $83.7 \%(81.4-86.0)$ with B and CT. 
After 200 deaths, no difference in OS was noted between the groups (HR 0.84, 95\% CI 0.64-1.12; $\mathrm{p}=0.23$ ). Use of B versus CT alone was associated with increased incidences of grade 3 or worse hypertension (154 (12\%) vs. 8 (1\%) patients), severe cardiac events occurring at any point during the 18 -month safety reporting period $(19(1 \%)$ vs. $2(<0.5 \%))$, and treatment (B, CT, or both) discontinuation $(256(20 \%)$ vs. $30(2 \%))$. B cannot be recommended as adjuvant treatment in unselected patients with TNBC. Further follow-up needs to assess the potential effect of B on OS.

Based on these data, the use of metronomic $\mathrm{X}$ may gain more interest due to its low toxicity profile and lower price tag.

The findings that a subset of TNBC is immunogenic and actively involves the immune system [25-28] provides a strong rationale for testing immunotherapies or drugs with an immunomodulatory mechanism of action. Different trials are ongoing to establish the role of immune checkpoint inhibitors alone or in combination, and of other immunotherapies or CT in TNBC.

Metronomic CT coupled with anticancer vaccines has also been investigated to determine whether this combination could be a promising approach.

Ongoing and planned studies in North America, such as the Eastern Cooperative Oncology Group-American College of Radiology Imaging Network (ECOG-ACRIN) EA1131 (NCT02445391) and SWOG (Southwest Oncology Group) S1418, are trying to define the role of X, platinum CT, or immune checkpoint inhibitors in patients with no pCR after neoadjuvant treatment. The EA1131 trial is randomizing patients to either $\mathrm{X}$ or carboplatin for 6 cycles.
The S1418/BR006 trial, conducted jointly by the Southwest Oncology Group (SWOG) and the National Surgical Adjuvant Breast and Bowel Project/Radiation Therapy Oncology Group/Gynecologic Oncology Group (NRG), is randomizing patients to observation or 1 year of pembrolizumab adjuvant therapy.

\section{Conclusion}

Further progress is needed to improve the outcome of TNBC patients in the adjuvant setting. Post-neoadjuvant strategies need further proof of benefit before becoming a standard approach. Low-dose maintenance therapy remains an attractive approach for additional studies in TNBC because of its low cost and good manageability and tolerability. Future plans should include a study evaluating low-dose oral maintenance for those TNBC patients who continue to have high-risk disease in the post-adjuvant and post-neoadjuvant setting.

\section{Disclosure Statement}

The authors certify that they have no affiliations with or involvement in any organization or entity with any financial interest (such as honoraria; educational grants; participation in speakers' bureaus; membership, employment, consultancies, stock ownership, or other equity interest; and expert testimony or patent-licensing arrangements), or non-financial interest (such as personal or professional relationships, affiliations, knowledge or beliefs) in the subject matter or materials discussed in this manuscript.

\section{References}

1 Trivers KF, Lund MJ, Porter PL, Liff JM, Flagg EW, Coates RJ, Eley JW: The epidemiology of triple-negative breast cancer, including race. Cancer Causes Control 2009;20:1071-1082.

2 Lin NU, Vanderplas A, Hughes ME, Theriault RL, Edge SB, Wong YN, Blayney DW, Joyce CN, Winer EP, Weeks JC: Clinico-pathologic features, patterns of recurrence, and survival among women with triplenegative breast cancer in the National Comprehensive Cancer Network. Cancer 2012;118:5463-5472.

3 Berry DA, Cirrincione C, Henderson IC, Citron ML, Budman DR, Goldstein LJ, Martino S, Perez EA, Muss HB, Norton L, Hudis C, Winer EP: Estrogen-receptor status and outcomes of modern chemotherapy for patients with node-positive breast cancer. JAMA 2006; 295:1658-1667.

4 Villarreal-Garza C, Khalaf D, Bouganim N, Clemons M, Peña Curiel O, Baez-Revueltas B, Kiss A, Kassam F, Enright K, Verma S, Pritchard K, Myers J, Dent R: Platinum based chemotherapy in triple-negative breast cancer. Breast Cancer Res Treat 2014;146:567-572.

5 Lehmann BD, Bauer JA, Chen X, Sanders ME, Chakravarrthy $A B$, Shyr Y, Pietenpol JA: Identification of human triple-negative breast cancer subtypes and preclinical models for selection of targeted therapies. J Clin Invest 2011;121:2750-2767.
6 Bron S, Henry L, Faes-van't Hull E, Turrini R, Vanhecke D, Guex N, Ifticene-Treboux A, Iancu EM, Semilietof A, Rufer N, Lehr HA, Xenarios I, Coukos G, Delaloye JF, Doucey MA: TIE-2-expressing monocytes are lymphangiogenic and associate specifically with lymphatics of human breast cancer. Oncoimmunology 2016;5:e1073882.

7 Liedtke C, Mazouni C, Hess KR, André F, Tordai A, Mejia JA, Symmans WF, Gonzales-Angulo AM, Hennessy B, Green M, Cristofanilli M, Hortobagyi GN, Pusztai L: Response to neoadjuvant therapy and longterm survival in patients with triple-negative breast cancer. J Clin Oncol 2008;26:1275-1281.

8 Von Minckwitz G, Untch M, Blohmer JU, Costa SD, Eidtmann H, Fasching PA, Gerber B, Eiermann W, Hilfrich J, Huober J, Jackisch C, Kaufmann M, Konecny GE, Denkert C, Nekljudova V, Mehta K, Loibl S: Definition and impact of pCR on prognosis after neoadjuvant chemotherapy in various intrinsic breast cancer subtypes. J Clin Oncol 2012;30:1796-1804.

9 Von Minckwitz G, Schneeweiss A, Loibl S, Salat C, Denkert C, Rezai M, Blohmer JU, Jackisch C, Paepke S, Gerber B, Zahm DM, Kümmel S, Eidtmann H, Klare P, Huober J, Costa S, Tesch H, Hanusch C, Hilfrich J, Khandan F, Fasching PA, Sinn BV, Engels K, Mehta K, Nekljudova V, Untch M: Neoadjuvant carboplatin in patients with triple-negative and HER-2 positive early breast cancer (GeparSixto; GBG 66): a randomised phase 2 trial. Lancet Oncol 2014;15:747-756.
10 Early Breast Cancer Trialists' Collaborative Group: Polychemotherapy for early breast cancer: an overview of the randomised trials. Lancet 1998;352:930-942.

11 Henderson JC, Berry D, Demeteri G, Cirrincione C, Goldstein L, Martino S, Ingle JN, Cooper MR, Canellos G, Borden E, Fleming G, Holland JF, Graziano S, Carpenter J, Muss H, Norton L: Improved disease-free (DFS) and overall survival (OS) from the addition of sequential paclitaxel $(\mathrm{T})$ but not from the escalation of doxorubicin (A) dose level in the adjuvant chemotherapy of patients (pts) with node positive primary breast cancer (BC). Proc Am Soc Oncol 1998; 17:abstr 390a.

12 Hanahan D, Bergers G, Bergsland E: Less is more, regularly: metronomic dosing of cytotoxic drugs can target tumor angiogenesis in mice. J Clinical Invest 2000; 105:1045-1047.

13 Klement G, Baruchel S, Rak J, Man S, Clark K, Hicklin DJ, Bohlen P, Kerbel RS: Continuous low-dose therapy with vinblastine and VEGF receptor-2 antibody induces sustained tumor regression without overt toxicity. J Clin Invest 2000;105:R15-R24.

14 Hirata S, Matsubara T, Saura R, Hirohata K, Tateishi $\mathrm{H}$ : Inhibition of in vitro vascular endothelial cell proliferation and in vivo neovascularization by low-dose methotrexate. Arthritis Rheum 1989;32:1065-1073.

15 Colleoni M, Rocca A, Sandri MT, Zorzino L, Masci G, Nolè, Peruzzotti G, Robertson C, Orlando L, Cinieri S, de Braud F, Viale G, Goldhirsch A: Low-dose oral methotrexate and cyclophosphamide in metastatic breast cancer: anti-tumor activity and correlation with vascular endothelial growth factor levels. Ann Oncol 2002;13:73-80. 
16 André N, Carré M, Pasquier E: Metronomics: towards personalized chemotherapy? Nat Rev Clin Oncol 2014; 11:413-431

17 Kerbel RS, Kamen BA: The anti-angiogenic basis of metronomic chemotherapy. Nat Rev Cancer 2004;4: 423-436.

18 Toi M, Lee SJ, Lee ES, Ohtani S, Im YH, Im SA, Lim SB, Yanagita Y, Takao S, Ohno S, Aogi K, Iwata H, Kim A, Sasano H, Yokota I, Ohaschi Y, Masuda N: A phase III trial of adjuvant capecitabine in breast cancer patients with HER-negative pathologic residual invasive disease after neoadjuvant chemotherapy (CRATEX, JBCRG-04). Cancer Res 2016;76(suppl):abstr S1-07.

19 Chen G, Guo Z, Liu M, Yao G, Dong J, Guo J, Ye C: Clinical value of capecitabine-based combination adjuvant chemotherapy in early breast cancer: a meta-analysis of randomized controlled trials. Oncol Res 2017DOI: 10.3727/096504017X14897173032733.

20 Alagizy HA, Shehata MA, Hashem TA, Abdelaziz KK, Swiha MM: Metronomic capecitabine as extend adjuvant chemotherapy in women with triple negative breast cancer. Hematol Oncol Stem Cell Ther 2015;8: 22-27.

21 Lluch A, Torrecillas L, Barrios CH, Bines J, Gomez H, Getulio J, Ruiz-Borrego M, de la Haba J, Torres R, Ruiz A. CIBOMA/2004-01: a randomized phase III trial assessing adjuvant capecitabine (X) maintenance therapy after standard chemotherapy for triple-negative early breast cancer (EBC). Eur J Cancer Suppl 2009;7:304.

22 Lluch A, Gomez H, Ruiz-Borrego M, Bines J, Llombart A, Ramos M: First safety data from a randomized phase III (CIBOMA 2004-01/GEICAM/2003-11) trial assessing adjuvant capecitabine maintenance therapy after standard chemotherapy for triple-negative breast cancer. Proc SABCS 2010;abstr P5-10-15.

23 Zhong-yu Yuan: Phase III study of adjuvant capecitabine metronomic chemotherapy in triple-negative operable breast cancer. clinicaltrials. gov/show/NCT01112826.

24 Cameron D, Brown J, Dent R, Jackisch C, Mackey J, Pivot X, Steger GG, Suter TM, Masaku T, Parmar M Laeufle R, Im YH, Romieu G, Harvey V, Lipatov O, Pienkowski T, Cottu P, Chan A, Im SA, Hall PS, Babuteishvilli-Pacaud L, Henschel V, Deurloo RJ, Pallaud C, Bell R: Adjuvant bevacizumab-containing therapy in triple-negative breast cancer (BEATRICE): primary results of a randomised, phase 3 trial. Lancet Oncol 2013;14:933-942.
25 Loi S, Dushyanthen S, Beavis PA, Salgado R, Denkert C, Savas P, Denkert C, Savas P, Combs S., Rimm DL, Giltnane JM, Estrada MV, Sanchez V, Sanders ME, Cook RS, Pilkinton MA, Mallal SA, Wang K, Miller VA, Stephens PJ, Yelensky R, Doimi FD, Gomez H, Ryzhov SV, Darcy $\mathrm{PK}$, Arteaga CL, Balko JM: RAS/MAPK activation is associated with reduced tumor-infiltrating lymphocytes in triple-negative breast cancer: therapeutic cooperation between MEK and PD1/PDL1 immune checkpoint inhibitors. Clin Cancer Res 2016;22:1499-1509.

26 Issa-Nummer Y, Darb-Esfahani S, Loibl S, Kunz G, Nekljudova V, Schrader I, Sinn BV, Ulmer HU, Kronenwett R, Just M, Kühn T, Diebold K, Untch M, Holms F, Blohmer JU, Habeck JO, Dietel M, Overkamp F, Krabisch P, von Minckwitz G, Denkert C: Prospective validation of immunological infiltrate for prediction of response to neoadjuvant chemotherapy in HER2-negative breast cancer - a substudy of the neoadjuvant GeparQuinto trial. PLoS One 2013;8:e79775.

27 Dieci MV, Criscitiello C, Goubar A, Viale G, Conte P, Guarneri V, Ficarra G, Mathieu MC, Delaloge S, Curigliano G, Andre F: Prognostic value of tumorinfiltrating lymphocytes on residual disease after primary chemotherapy for triple-negative breast cancer: a retrospective multicenter study. Ann Oncol 2014;25: 611-618.

28 Brown SD, Warren RL, Gibb EA, Martin SD, Spinelli JJ, Nelson BH, Holt RA: Neo-antigens predicted by tumor genome meta-analysis correlate with increased patient survival. Genome Res 2014;24:743-750.

29 Ludwig Breast Cancer Study Group: Combination adjuvant chemotherapy for node-positive breast cancer: inadequacy of a single peri-operative cycle. N Engl J Med 1988;319:677-683.

30 Ludwig Breast Cancer Study Group: Prolonged disease-free survival after on course of perioperative adjuvant chemotherapy for node-negative breast cancer. $\mathrm{N}$ Engl J Med 1989;320:491-496.

31 Colleoni M, Litman HJ, Castiglione-Gertsch M, Sauerbrei W, Gelber RD, Bonetti M, Coates AS, Schumacher M, Bastert G, Rudenstam CM, Schmoor C, Lindtner J, Collins J, Thurlimann B, Holmberg SB, Crivellari D, Beyerle C, Neumann RLA, Goldhirsch A; for the International Breast Cancer Study Group and the German Breast Cancer Study Group: Duration of adjuvant chemotherapy for breast cancer: a joint analysis of two randomized trials investigating three versus six courses of CMF. Br J Cancer 2002;86:1705-1714.
32 Saurerbrei W, Bastert G, Bojar H, Beyerle C, Neumann RL, Schmoor C, Schumacher M: Randomized $2 \times 2$ trial evaluating hormonal treatment and the duration of CT in node-positive BC patients: an update based on 10 years' follow up. J Clin Oncol 2000;18:94-99.

33 International Breast Cancer Study Group: Duration and reintroduction of adjuvant chemotherapy for node-positive premenopausal breast cancer patients. J Clin Oncol 1996;14:1885-1894.

34 Fischer B, Brown AM, Dimitrov NV, Poisson R, Redmond C, Margolese RG, Bowman D, Wolmark N, Wickerham DL, Kardinal CG: Two months of doxorubicin-cyclophosphamide with and without interval reinduction therapy compared with 6 months of cyclophosphamide, methotrexate, and fluoruracil in positive-node breast cancer patients with tamoxifen-non responsive tumors: results from the National Surgical Adjuvant Breast and Bowel Project B-15. J Clin Oncol 1990;8:1483-1496.

35 Clahsen PC, van de Velde CJ, Welvaart K, van Driel OJ, Sylvester RJ: Ten-year results of a randomized trial evaluating prolonged low-dose adjuvant chemotherapy in node-positive breast cancer: a joint European Organization for Research and Treatment of CancerDutch Breast Cancer Working Party study. J Clin Oncol 1995;13:33-41.

36 Nasr KE, Osman MA, Elkady MS, Ellithy MA: Metronomic methotrexate and cyclophosphamide after carboplatin included adjuvant chemotherapy in triple negative breast cancer: a phase III study. Ann Transl Med 2015;3:284-294.

37 Colleoni M, Gray K P, Gelber S, Làng I, Thurlimann B, Gianni L, Abdi Ehtesham AA, Gomez HL, Linderholm BK, Puglisi F, Tondini C, Kralidis E, Eniu A, Cagossi K, Rauch D, Chirgwin J, Gelber RD, Regan MM, Coates AS, Price KN, Viale G, Golhirsch A: Low-dose oral cyclophosphamide and methotrexate maintenance for hormone receptor-negative early breast cancer: International Breast Cancer Study Group Trial 22-00. J Clin Oncol 2016;34:3400-3408. 